\title{
Effect of dispersion on the reflectivity of an asymmetric Fabry-Pérot étalon
}

\author{
J. F. Hefferman a) and J. Hegarty \\ Department of Physics, Trinity College, Dublin 2, Ireland
}

(Received 23 August 1994; accepted for publication 28 November 1994)

\begin{abstract}
We have shown that the spectral properties of an asymmetric Fabry-Pérot étalon, with a multiple quantum well cavity, are very sensitive to dispersion in the refractive index. We also show that this effect can be used to measure the dispersion around the exciton absorption features in the $\mathrm{GaAs} / \mathrm{Al}_{0.35} \mathrm{Ga}_{0.65} \mathrm{As}$ multiple quantum well. (C) 1995 American Institute of Physics.
\end{abstract}

Epitaxially grown multiple quantum well structures have been used extensively in a wide variety of optoelectronic applications since their first appearance almost 20 years ago. ${ }^{1}$ In particular there is a variety of devices which rely on the resonant cavity effects of a Fabry-Péot étalon to enhance the excitonic nonlinear optical and electro-optical properties of quantum wells. ${ }^{2}$ In most cases multiple quantum wells are grown between two dielectric stack mirrors in an integrated étalon structure. Optical modulation or optical switching is achieved by utilizing the changes in reflectivity or transmission of the étalon due to induced variations in absorption and/or refractive index in the quantum wells. Such variations can be achieved by a number of different methods. The presence of a high density of optically generated free carriers bleaches the strong exciton absorption resonances of the quantum wells and is the basis of a number of all-optical devices. ${ }^{3-5}$ Alternatively application of an electric field perpendicular to the plane of the wells leads to a rigid shift of the band-edge absorption (the quantum confined Stark effect) and this effect is used extensively in electro-optic devices. ${ }^{6}$

In both all-optical and electro-optical devices the étalon design is similar. The multiple quantum well forms part of the spacer, or cavity, between two epitaxially grown Bragg stack mirrors. This forms a Fabry-Pérot étalon and the quantum wells are chosen so that their absorption edge is close to a resonant wavelength of the étalon, which is determined by the overall thickness of the cavity. Since both the exciton resonance and the Fabry-Pérot resonance are spectrally narrow there are severe constraints on the positioning of them with respect to each other. The exciton resonance is determined by the quantum well thickness and composition and is now well controlled in epitaxial growth. Small variations in quantum well thickness generally do not affect the energy of the exciton transitions except in the case of very narrow wells. The Fabry-Pérot resonance, on the other hand, is particularly sensitive to the spacer thickness $d$ and its refractive index $n$ through the resonance condition, $m \lambda=2 \mathrm{nd}$. Here $m$ is the order of the Fabry-Pérot resonance and $\lambda$ is the free space wavelength. The sharp exciton resonances in the multiple quantum well leads to significant index dispersion which must be taken into account in the design of devices. In this letter we show that linear dispersion can have a large effect on the spectral properties of a Fabry-Pérot étalon and further that we can use these effects to directly calculate the dispersion. Taking dispersion into account in device design

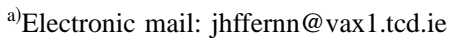

will be increasingly important as materials quality and wafer uniformity improves.

The structure we have investigated is an epitaxially grown asymmetric Fabry-Pérot étalon. The étalon is asymmetric in that it has a high reflectivity back mirror and a lower reflectivity front mirror. It was grown by metalorganic vapor phase epitaxy (MOVPE) on a semi-insulating GaAs substrate. Closest to the substrate is a quarter wavelength $(\lambda / 4)$ dielectric stack mirror consisting of 14.5 periods of alternating layers of $\mathrm{AlAs}$ and $\mathrm{Al}_{0.1} \mathrm{Ga}_{0.9} \mathrm{As}$. The thicknesses were chosen to be $(\lambda / 4)$ at $850 \mathrm{~nm}$ and the mirror has a reflectivity of $96 \%$ from 810 to $880 \mathrm{~nm}$. On top of this 73 periods of $95 \AA \mathrm{GaAs}$ wells and $69 \AA \mathrm{Al}_{0.3} \mathrm{Ga}_{0.7} \mathrm{As}$ barriers were grown. The étalon was completed by the front mirror which is simply the $30 \%$ reflectivity of the air/multiple quantum well interface. The nonlinear optical properties of a similar structure have been reported before. ${ }^{3}$ The well width was designed for a heavy hole exciton resonance at $850 \mathrm{~nm}$. The absorption curve of Fig. 1 shows that the exciton is at this wavelength and in fact there is a negligible shift of the peak across the entire 2 in. wafer. The number of quantum wells was chosen so that the absorption in the low energy tail of the exciton resonance, at around $853 \mathrm{~nm}$, is enough to give a low reflectivity at a coincident Fabry-Pérot

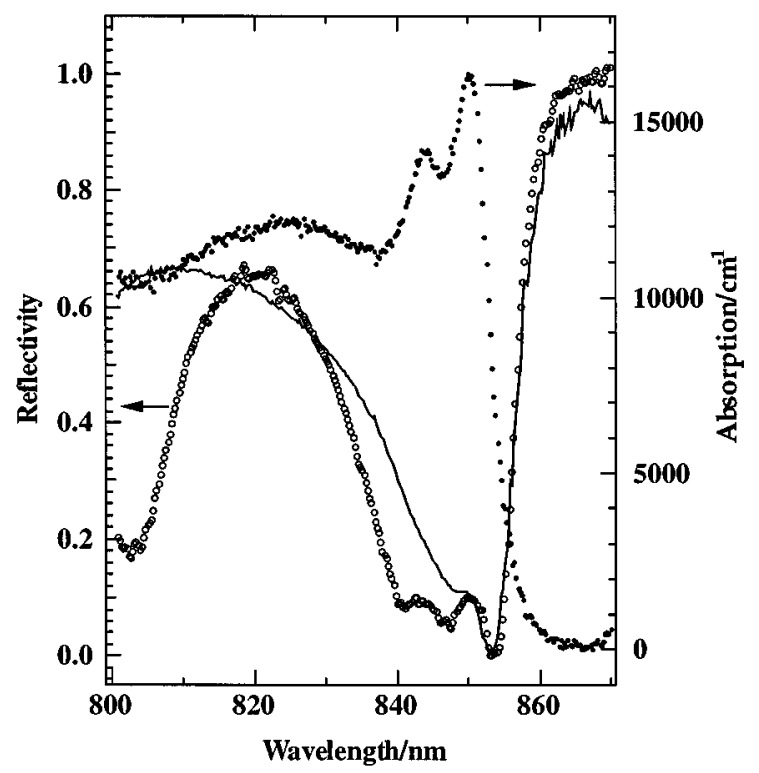

FIG. 1. Reflectivity spectrum (open circles) of asymmetric Fabry-Pérot étalon and absorption spectrum of multiple quantum well (full circles). Also shown (line) is a calculated reflectivity spectrum based on a single value of refractive index and the above absorption spectrum. 
resonance. ${ }^{3}$ The barrier thickness was then chosen so that the total thickness of the cavity gives a Fabry-Pérot resonance around $853 \mathrm{~nm}$. The required thickness obviously depends on the value chosen for the refractive index of the cavity. As a first approximation the refractive index was taken as an averaged literature value for the well and barrier constituents. ${ }^{7,8}$ Even if this value is not accurate the nonuniformity in the growth usually means that a number of positions can be found on the wafer where the optical thickness of the cavity is correct and consequently the band edge and étalon resonance are appropriately positioned. Although nonuniformity is helpful for research purposes this is not the case if real devices are to be produced since other areas of the wafer are either neglected or must be subjected to a number of postgrowth treatments, such as etching ${ }^{9}$ or layer disordering, ${ }^{10}$ in order to correct the cavity.

A reflectivity spectrum of the étalon is shown in Fig. 1 with the corresponding absorption spectrum of the quantum well. All measurements were carried out on a piece of the wafer wit area approximately $4 \mathrm{~mm}^{2}$. This ensures that all quantities such as absorption and cavity thickness are from the same position on the wafer and that there is no error due to wafer nonuniformity. Reflectivity and absorption measurements were made with an argon ion-pumped titaniumsapphire laser and synchronous detection. Reflectivities were normalized to that of a broadband high reflectivity infrared mirror. The absorption spectrum was obtained from a portion of the sample which had been antireflection coated by thermal evaporation of a $\lambda / 4$ layer of $\mathrm{SiO}$. This coating reduces the front mirror reflectivity to less than $1 \%$ around the exciton wavelength and less than $5 \%$ over a range of about 50 $\mathrm{nm}$. With such a low top surface reflectivity, Fabry-Pérot effects can be neglected and the absorption may be obtained by measuring the incident and reflected powers. To measure the dispersion of the refractive index we needed to measure the physical thickness of the cavity. The multiple quantum well was etched off using a standard wet etch on a masked piece of the wafer. The etch was highly selective for AlAs which is the first layer of the mirror, so we expect that only the quantum wells were removed. The thickness of the cavity was measured at various points on the etched surface using a Tencor step height instrument with a resolution of approximately $50 \AA$. The measured thickness varied somewhat across the etched area with an average value of (1.180 $+0.008) \mu \mathrm{m}$. The error in this number is the variation in the etch depth and not the variation in the actual thickness of the as-grown sample. This is clear from the spectral characteristics around the etched piece which show little variation in the Fabry-Pérot resonance position. The reflectivity spectrum in Fig. 1 was then measured at a point near the etch and finally this same point was antireflection coated and the absorption spectrum of Fig. 1 measured.

Figure 1 shows that the reflectivity spectrum is complicated and cannot be simply explained by the shape of the absorption spectrum. There are three dips in reflectivity which do not coincide with the peaks in the absorption spectrum due to the light and heavy hole excitons. The reflectivity at $853 \mathrm{~nm}$ is very close to zero and can only be explained by the fact that this is a Fabry-Pérot resonance and that the absorption strength is such that the effective reflectivity of the back mirror is equal to that of the front mirror. The cavity is then said to be impedance matched at this wavelength. The absorption at the exciton peaks is too strong which explains why the reflectivity is higher at these wavelengths. To show that the reflectivity spectrum is not simply due to the structure in the absorption spectrum we calculated the reflectivity of the etalon with a single value for the refractive index. All calculations of étalon reflectivity used a full transfer matrix approach $^{11}$ to account for the wavelength dependent phase contribution that occurs in the Bragg stack mirror. With the measured value for the cavity thickness we chose a value for the refractive index of the cavity so that the resonance condition was satisfied at $853 \mathrm{~nm}$. The rest of the spectrum was then calculated using the absorption spectrum of Fig. 1 and this single value for the refractive index. The solid line in Fig. 1 is the result of this calculation and it shows that this approach does not reproduce the experimental spectrum. The calculated and experimental spectra agree around $853 \mathrm{~nm}$ as we have chosen the refractive index to be correct for this wavelength. However the curves disagree at other wavelengths with differences in reflectivity of up to $20 \%$. We attribute the discrepancy to the dispersion in the refractive index of the cavity. The Fabry-Pérot resonance condition, $m \lambda=2$ nd, is thus satisfied at more than one wavelength for a given order $m$. The result shows that the spectral properties of the Fabry-Pérot étalon are significantly affected by dispersion in the cavity. The actual dependence of the reflectivity on the dispersion is a function of the particular FabryPérot structure but it is clear that it has a large effect in this particular étalon which is typical of a number of electro-optic devices. ${ }^{6}$

To confirm that the spectral properties of the FabryPérot are determined by the dispersion of the refractive index, we have used the experimentally determined reflectivity and absorption spectra to measure this dispersion. Having measured the cavity thickness, we use the transfer matrix approach to calculate the reflectivity at each wavelength using the measured value of the absorption at this wavelength. The value for the refractive index used was then varied until the calculated and experimental reflectivity agreed. This procedure was used at each wavelength where the absorption spectrum was valid. The wavelength range for calculation was limited on the short wavelength side by the range over which the absorption spectrum is valid, which is determined by the antireflection coating. To extend this we would have to deposit a multilayer antireflection coating. We also could not extend the measurements beyond $860 \mathrm{~nm}$ because this is in the "off resonance" region of the spectrum where the lack of absorption and high reflectivities mean that the spectrum is not very sensitive to variations in refractive index.

The calculated refractive index is shown in Fig. 2 superimposed on the absorption spectrum of the quantum wells. Since the optical thickness of the cavity is the important parameter, the error on the absolute value of the refractive index is primarily the error in the physical thickness, i.e., about $0.7 \%$. However, the shape of the curve is independent of this and shows that around the heavy hole exciton wavelength there is a pronounced peak in refractive index: ap- 


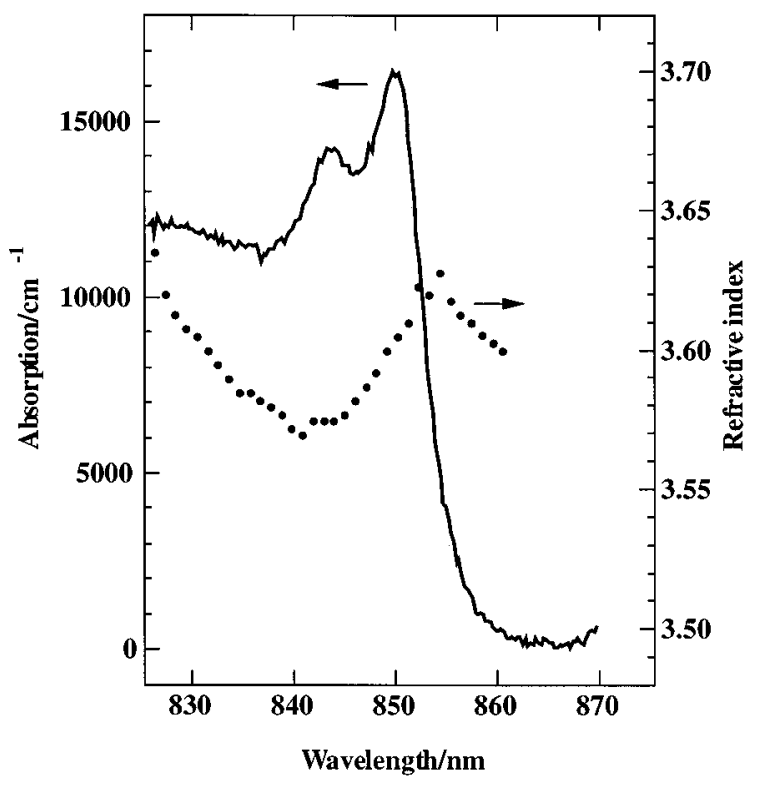

FIG. 2. Band-edge refractive index of multiple quantum well (circles) showing peak around the heavy hole exciton absorption at $850 \mathrm{~nm}$. The absorption spectrum of the quantum well is also shown (line).

proximately $1.5 \%$ higher than the background trend seen between 830 and $840 \mathrm{~nm}$. This value and shape are as expected from a Kramers-Kronig analysis and from some independent measurements of exciton dispersion in quantum wells. $^{12,13}$ The few previous measurements of dispersion have used multiple quantum wells embedded in a waveguide structure. The coupling to such a waveguide depends on the refractive index of the multiple quantum well. Such measurements and those presented here are the only ones we are aware of that directly measure this technologically important material parameter.

Figure 3 shows the experimental and calculated reflectivity spectrum of the étalon. The good agreement between them verifies that the complicated structure of the spectrum is due to the combination of the strong changes in absorption and the refractive index around the excitonic absorption features of the quantum wells. This technique may be extended to measure the effect of narrower exciton linewidths on the dispersion and in particular the effect of the light hole exciton, which is not resolved in this structure due to the strong overlap between light and heavy hole excitons in absorption.

In conclusion, we have shown that the reflectivity spectrum of an asymmetric Fabry-Pérot étalon, with a multiple quantum well cavity, is significantly affected by refractive index dispersion. This dispersion arises due to the strong absorption features in the quantum wells associated with quantum confined excitons. We have further shown that the

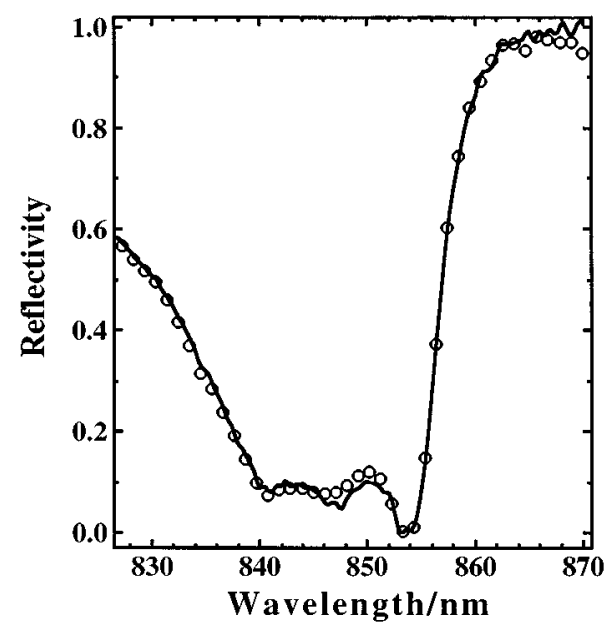

FIG. 3. Measured (line) and calculated (circles) reflectivity of asymmetric Fabry-Pérot étalon. The calculation includes the measured absorption and refractive index of the multiple quantum well that forms the cavity of the étalon.

dispersion in the quantum wells can be measured by fitting the reflectivity spectrum of the étalon and find that, in the low energy tail of the heavy hole exciton absorption, the refractive index increases by $1.5 \%$ from the background trend. These results are expected to be of increasing importance in étalon design as tolerances on growth accuracy and nonuniformity improve.

The authors wish to thank J. S. Roberts for providing us with the sample and B. Corbett for the cavity thickness measurements.

${ }^{1}$ C. Weisbuch and B. Vinter, Quantum Semiconductor Structures (Academic, London, 1991).

${ }^{2}$ H. M. Gibbs, Optical Bistability: Controlling Light with Light (Academic, New York, 1985).

${ }^{3}$ J. F. Heffernan, M. H. Moloney, J. Hegarty, J. S. Roberts, and M. Whitehead, Appl. Phys. Lett. 58, 2877 (1991).

${ }^{4}$ B. G. Sfez, J. L. Oudar, J. C. Michel, R. Kuszeleviscz, and R. Azoulay, Appl. Phys. Lett. 57, 324 (1990).

${ }^{5}$ M. H. Moloney J. F. Heffernan, J. Hegarty, R. Grey, and J. Woodhead, Appl. Phys. Lett. 63, 435 (1993).

${ }^{6}$ See, for example, special issue Electro-optic Modulation of Light, edited by M. Whitehead, Opt. Quantum Electron. 25 (1993)

${ }^{7}$ D. E. Aspnes, S. M. Kelso, R. A. Logan, and R. Bhat, J. Appl. Phys. 60, 754 (1986).

${ }^{8}$ O. Madelung, Semiconductors, Group IV Elements and II-V Compounds, Data in Science and Technology (Springer, New York, 1991).

${ }^{9}$ H. Kezhong, R. Cartland, L. Chen, K. Kaviani, P. Chen, and A. Madhukar, Optical Society of America Topical meeting on Spatial Light Modulators and Applications, Palm Springs, CA, 1993 (unpublished) paper SWB2-1.

${ }^{10}$ J. H. March, Semicond. Sci. Technol. 8, 1136 (1993).

${ }^{11}$ M. Born and E. Wolf, Principles of Optics (Pergamon, Oxford, 1980).

${ }^{12}$ K. B. Kahen and J. P. Leburton, Appl. Phys. Lett. 49, 734 (1986).

${ }^{13}$ G. J. Sonek, J. M. Ballantyne, Y. J. Chen, G. M. Carter, S. W. Brown, E. S. Koteles, and J. P. Salerno, Proceedings of the 18th Topical Meeting on Integrated and Guided Wave Optics, Kissimee, FL (IEEE Optical Society of America, Washington, DC 1986). 